\title{
PROPUESTA DE INTERVENCIÓN PARA DETENER EL BULLYING
}

\section{INTERVENTION PROPOSAL TO STOP BULLYING}

\author{
Eneko Tejada Garitano' \\ Ainara Romero Andonegi \\ Urtza Garay Ruiz \\ Universidad del País Vasco, España
}

\section{RESUMEN}

El bullying es un tipo de violencia específica que sucede en el ámbito educativo y que tiene una gran repercusión social. Los factores de riesgo que contribuyen a que se produzca acoso escolar son múltiples. A pesar de que los expertos recomiendan abordar el problema desde una perspectiva psicosocial y sistémica, las instituciones educativas tienden a intervenir solo sobre los factores personales de la figura de los acosadores, por lo que hay que optimizar las acciones que contribuyen a detener el bullying. A pesar de que existen diferentes instrumentos para prevenir las conductas de acoso escolar, los centros educativos no disponen de tantos medios para detener esas conductas cuando ya se han producido. El objetivo de la investigación que se presenta a continuación es analizar la actuación llevada a cabo con el fin de detener las conductas bullying y diseñar un procedimiento específico que permita abordar el problema. Se ha utilizado una metodología cualitativa de investigación acción, a través de la que se han categorizado las intervenciones llevadas a cabo para detener las conductas de acoso. De los resultados de la investigación se extrae un procedimiento secuenciado en fases que constituye una propuesta

\footnotetext{
${ }^{1}$ Correspondencia: Eneko Tejada Garitano: Barrio Sarriena, S/N, Leioa. 48940-Vizcaya. Correo-e:
} eneko.tejada@ehu.eus 
válida para intervenir con alumnado acosador ya que contribuiría a detener las conductas de acoso escolar.

Palabras clave: bullying, detención del acoso, intervención antibullying, coaching, psicopedagogía

\section{ABSTRACT}

Bullying is a specific type of violence that happens in education and has a great social impact. There are multiple risk factors that contribute to harassment being produced at school. Although experts recommend tackling the problem from a psychosocial and systemic perspective, educational institutions tend to take action by just dealing with the personal issues that lead bullies to have such behavior. Therefore, actions that help stop bullying have to be optimized. Although there are different mechanisms and tools to prevent bullying behavior, schools do not count on so many means to prevent that kind of behavior from happening. The aim of the present research is to analyze the actions which are carried out in order to stop harassment at school and design a specific procedure to tackle and give an answer to the problem. A research-action qualitative methodology has been used, in which the interventions carried out to stop bullying behavior have been categorized. By analyzing the outcome of the investigation, we can work out a procedure which is sequenced in phases. This proposal is valid to take action on harassers due to the fact that it will prevent bullying behavior from happening.

Key Words: bullying, stop bullying, antibullying intervention, coaching, psychopedagogy

\section{Introducción}

El Bullying es un fenómeno de gran repercusión social que hace referencia a un tipo de agresión (Cerezo y Méndez, 2013; Espelage y Swearer, 2003; Olweus, 1993) o violencia específica que sucede en el ámbito educativo (Álvarez-García, Núñez, Rodríguez, Álvarez y Dobarro, 2011). Este problema del que se han realizado innumerables investigaciones (Zych, Ortega-Ruiz y Del Rey, 2015), se ha detectado en países tan diversos como Finlandia, Reino Unido, México, Brasil, Japón, etc. (Garaigordobil y Oñederra, 2010a).

Fundamentalmente se trata de un conjunto de acciones dañinas que uno o varios sujetos causan de forma consciente, repetida y mantenida en el tiempo (Avilés, 2013; Olweus, 1993) con objeto de causar daño a un igual que se encuentra en una situación de indefensión física, psicológica y/o social (Cerezo, Calvo y Sánchez, 2011; Piñuel y Oñate, 2007).

Los factores de riesgo que contribuyen a que se produzca el bullying son múltiples y diversos (Cassiani-Miranda, Gómez-Alhach, Cubides-Munévar y Hernández-Carrillo, 2014; Einarsen y Hauge, 2006), por lo que su prevención y erradicación puede realizarse desde diferentes vertientes.

De acuerdo con lo señalado, algunos expertos (Garaigordobil y Oñederra, 2010b) recomiendan abordar el problema desde una perspectiva psicosocial y sistémica de forma que involucre y afecte al conjunto de la comunidad educativa (Della Cioppa, O'Neil y Craig, 2015; Ferrel, Cuan, Londoño y Ferrel, 2015). A pesar de ello, hay instituciones educativas que solo intervienen y permiten actuar sobre los factores personales de la figura del agresor, aún a riesgo 
de que se incrusten los patrones de acoso en su cultura escolar (Suckling y Temple, 2006). Es por ello que con objeto de optimizar las acciones que se pueden realizar en los centros con los acosadores, se deben identificar bien los rasgos y componentes personales de estos maltratadores que repercuten tanto en la aparición como mantenimiento del bullying.

Los alumnos que maltratan en la escuela siempre tienen un motivo. Pueden ser más o menos conscientes de la razón por la que agreden, pero detrás de su conducta siempre hay una causa que les reporta algún tipo de beneficio social, psicológico o material (Perren y Alsaker, 2006). Para ello, actúan manipulando situaciones sociales (Romera, Cano, García-Fernández y OrtegaRuiz, 2016) o ejerciendo la agresión directa bajo el beneplácito de otros iguales (Berger y Caravita, 2016).

La ganancia que experimentan contribuye a reforzar su comportamiento proactivo agresor (Bender y Lösel, 2011; Hubbard, McAuliffe, Morrow y Romano, 2010), a creer efectivamente que son buenos agrediendo y a alimentar su expectativa de lograr un resultado positivo respecto a uno negativo (Andreu, Ramírez y Raine, 2006; Arsenio, Gold y Adams, 2004; Hawley, Stump y Ratliff, 2010), lo que les conduce a sobrevalorar el efecto de su conducta (Marsee y Frick, 2007).

El alumno que agrede en estos términos, cuando percibe que las ventajas de acosar son mayores que los inconvenientes, tiende a comportarse de forma más agresiva que el que siente que puede ser castigado (Gómez et al., 2005) por lo que es importante conseguir que no logre el objetivo que persigue, es decir las ganancias instrumentales (Valdés Cuervo, Yáñez, Quijada y Martínez, 2013).

Otros acosadores, en cambio, actúan en clave reactiva. Agreden como resultado de un hipotético sentimiento de amenaza que no se ha producido. Es decir, sufren una baja comprensión de los sentimientos y emociones de las personas con las que interactúan como consecuencia de una pobre regulación a la respuesta de estímulos ambiguos, (Gini, Pozzoli y Hauser, 2011; Vitaro, Brendgen y Tremblay, 2002). De esta forma, realizan un sesgo atributivo hostil a la hora de procesar la información (Berger, 2006) que les lleva a interpretar de forma errónea las señales sociales y a percibir como intencional un daño que no lo es (Hubbard et al., 2001).

Los alumnos que muestran este tipo de conductas deben ser entrenados en percibir y decodificar la información social correctamente (Smithmyer, Hubbard y Simons, 2000). Y es que desarrollar las habilidades de pensamiento causal, alternativo y consecuencial, les permitiría a los agresores descifrar la información que proviene de las diferentes situaciones sociales, imaginar soluciones alternativas y prever las consecuencias de las acciones antes de realizarlas (Segura, 2005). Además contribuiría a que no entendiesen el medio escolar en clave dicotómica de víctima o agresor.

Otro tipo de factor de riesgo personal se encuentra vinculado a las conductas de interacción social inapropiadas. De esta forma, algunos alumnos agreden porque utilizan estrategias de afrontamiento no válidas para resolver las situaciones del día a día debido a un desfase entre la transacción de su demanda interna y de lo que requiere el ambiente o situación (Mestre et al., 2012; Gázquez, Pérez y Carrión, 2011). Es conviene que los alumnos controlen esta impulsividad, que por otra parte es predictora de conductas delictivas (Jiménez-Barbero, Ruiz-Hernández, Velandrino-Nicolás y Llor-Zaragoza, 2016) y violentas en general (López, Domínguez y Álvarez, 2010; Vera, 2010), ya que favorece al aumento de comportamientos dañinos (Andreu, Peña y Ramírez, 2009).

Algunos de estos acosadores atribuyen a otros la autoría de las agresiones que se han producido en contexto escolar (Dueñas y Senra, 2009), por lo que desde el ámbito educativo se ha de ayudar a que realicen una atribución causal interna de los acontecimientos (Avilés, 2006), a que se responsabilicen de sus acciones y a que entiendan que su conducta produce unos 
resultados que tienen unas consecuencias para ellos (Suckling y Temple, 2006). Entre las estrategias orientadas a tal fin se encuentra la aplicación de las normas de convivencia que tienen los centros educativos (Antelo, Cagide, Cruz y Buceta, 2010), el entrenamiento en la autoobservación y autorregulación, y el desarrollo de las habilidades sociales (Cerezo, 2006).

La influencia de la inteligencia emocional en el bullying es otro de los aspectos que los diferentes estudios analizan. Algunos autores (Pelegrín y Garcés de Los Fayos, 2008; Zimmerman, 2005) señalan que los alumnos agresores muestran deficiencias en el desarrollo de la empatía, entendida desde una dimensión afectiva y cognitiva (Bush, Mullis y Mullis, 2000). De esta forma un acosador con una empatía negativa es capaz de detectar el momento de mayor debilidad de su víctima, agredirla y no mostrar ningún sentimiento de culpa (Cerezo, 2009; Ortega y Mora-Merchán, 2008).

Se debe prestar especial atención a este tipo de manifestaciones de insensibilidad emocional (Frick et al., 2003), ya que tradicionalmente se ha relacionado con el desarrollo de la personalidad psicopática (Romero, Triñanes y Iglesias, 2011). Trabajar la capacidad de situarse en el lugar de los demás, así como ayudar al alumnado a comprender sus sentimientos y emociones contribuiría a que se cometiesen menos agresiones (Gutiérrez, Escartí y Pascual, 2011) y favorecería la convivencia (Martorell, González, Rasal y Estellés, 2009).

También hay que prestar especial atención a la gran frustración que sienten algunos alumnos a la hora de valorar su vida (Martínez, Buelga y Cava, 2007), ya que expían su dolor causando daño a otros. El hecho de que algunos alumnos no acepten su status social, su situación personal, familiar, etc. o simplemente no posean un objeto material deseado, hace que proyecten su impotencia en forma de agresión física psicológica y/o social sobre los compañeros o iguales que representan su anhelo (Cerezo 2009; González-Ortega, Echeburúa y Corral, 2008).

La autoestima de los acosadores también es un rasgo personal que ha sido ampliamente investigado en el bullying y del que se han obtenido resultados contradictorios. A este respecto algunos trabajos evidencian que los maltratadores escolares tienen un nivel alto de autoestima (Estévez, Herrero, Martínez y Musitu, 2006; Díaz-Aguado, 2004), y otros por el contrario bajo (Hanish y Guerra, 2004). En cualquiera de los casos parece más correcto señalar que los acosadores escolares tienen un tipo de autoestima alta e inestable (Kernis, Grannemann y Barclay, 1989), condicionada por la percepción de seguridad que tienen de sí mismos, de su independencia con respecto a las valoraciones de los demás, y de su empatía (Coover y Murphy, 2000). Así los alumnos que buscan reconocimiento social para reforzar su autoestima tienen más facilidad para desarrollar comportamientos agresivos (Rodkin, Ryan, Jamison y Wilson, 2013).

Los centros educativos disponen de numerosos recursos de carácter preventivo universal e integral (Greenberg, 2010; Vreeman y Carroll, 2007) en forma de programas y guías (Garaigordobil y Oñederra 2010a) que inciden sobre los factores de riesgo señalados. Para los contextos educativos en que la probabilidad de que se produzca bullying es más alta existen instrumentos más específicos y selectivos (Avilés, 2013; Beane, 2006; Weare y Nind, 2011). En menor proporción también hay métodos y técnicas como los que plantean Cerezo, Calvo y Sánchez, (2011) o Suckling y Temple (2006) para cuando el acoso escolar ya se ha producido, lo que hace que se utilicen recursos menos pertinentes como el arbitraje o la mediación que tienen su origen en la resolución de conflictos. De esta forma, disponer de un instrumento orientado a actuar sólo con los agresores y que permita parar sus conductas acosadoras tiene una importancia sustancial.

El trabajo que se presenta a continuación tiene por objeto analizar el conjunto de actuaciones que se han llevado a cabo para detener las conductas bullying de tres estudiantes y diseñar un instrumento que permita detenerlo en cualquier contexto educativo. 


\section{Método}

El estudio se llevó a cabo con tres alumnos del primer ciclo de Educación Secundaria Obligatoria (ESO) (15 años) que presentaban conductas de acoso escolar. Uno de los estudiantes pertenecía a un centro educativo público y los otros dos a privados concertados.

La detección de los estudiantes que realizaban acoso escolar se realizó ofreciendo a diversos centros de Educación Secundaria Obligatoria la posibilidad de recibir ayuda para dar respuesta a los posibles casos de bullying que pudiesen detectar. Los tres centros que solicitaron la ayuda accedieron a que se actuase con sus estudiantes a condición de que no se interviniese a través del protocolo antibullying del Departamento de Educación de la Comunidad Autónoma Vasca. En base a otras experiencias este recurso era percibido por los directores de los centros educativos como un instrumento que burocratiza el problema.

Los comportamientos de los estudiantes fueron catalogados como conductas agresivas susceptibles de ser tipificadas como acoso escolar. Esto permitió llevar a cabo un conjunto de actuaciones teniendo en cuenta lo que prescribe en el capítulo 3 del decreto de derechos y deberes (Gobierno Vasco. Decreto 201/2008, de 2 de diciembre), vías alternativas para la corrección de conductas, donde se especifica la posibilidad de llevar a cabo medidas educativas sin procedimiento en la medida en que estas no sean de acoso escolar.

De esta forma, se comunicó a los padres de estos estudiantes que se iba a llevar una actuación psicopedagógica como consecuencia de haber realizado unas conductas agresivas, que podían catalogarse como propias del acoso escolar.

De esta forma, se ha llevado a cabo un estudio de carácter cualitativo por medio del método de investigación-acción de espirales (McKernan, 1999) o ciclos (Elliott, 1993), que ha permitido tener una comprensión global, descriptiva e interpretativa de lo que ha sucedido en las diferentes intervenciones que se han llevado a cabo con los estudiantes acosadores.

El procedimiento de la investigación realizada consta de un ciclo completo compuesto por cuatro momentos o pasos. En el primero se planificó y configuró la secuencia de intervenciones que debía contribuir a detener las conductas de acoso que se estaban produciendo. Para ello, se tuvieron en cuenta las diferentes teorías sobre los factores personales que contribuyen a la aparición del bullying y las aportaciones de los tutores y orientadores de cada uno de los centros en las que estaban los acosadores.

A través de diferentes intervenciones, se diseñó una sucesión de acciones que tenían por objeto que los estudiantes no obtuviesen ningún beneficio o rentabilidad de su actividad acosadora, que reconociesen y asumiesen su responsabilidad, y que identificasen las conductas de acoso que habían cometido. También se planificaron las intervenciones que tenían como fin conocer la causa por la que los estudiantes agredían a sus compañeros, y las medidas específicas que podían contribuir a detenerlas.

En el segundo paso de este estudio, se llevaron adelante las intervenciones planificadas de forma metódica y reflexiva. Del mismo modo en este periodo, con el fin de conocer el efecto de las intervenciones realizadas por el educador externo con los acosadores, al inicio de cada sesión se plantearon unas preguntas control a los estudiantes agresores respecto a su conducta y actitud, para contrastarla con las observaciones de los profesores y con la información de la propia víctima que también fue recogida por el tutor. 
En un tercer momento de esta investigación, en el paso de observación, el instrumento de análisis de datos Atlas.ti (versión 22) permitió codificar y categorizar las respuestas que dieron los alumnos acosadores a las intervenciones del educador externo y representar la evolución del desvanecimiento de las conductas de acoso que fueron agrupadas en nueve tipos de agresiones.

Por último, en el cuarto paso, como resultado de la reflexión llevada a cabo de los datos obtenidos y la categorización realizada, se identificaron los momentos y fases por las que pasan los acosadores antes de dejar de agredir, lo que ha dado lugar al diseño de una nueva propuesta de actuación enriquecida y mejorada.

Se realizaron seis sesiones de aproximadamente una hora de duración. En cada una de ellas se llevaba a cabo una actividad cognitiva específica denominada ejercicio situacional que permitía a los acosadores adquirir un nuevo conocimiento en relación al bullying, y un ejercicio razonamiento que contribuía a la reflexionasen sobre su situación personal respecto al maltrato que estaban produciendo. La secuencia de los ejercicios cognitivos y razonamientos se estructuró en diferentes grados de complejidad.

Los instrumentos utilizados para recoger los datos sobre la incidencia de las intervenciones del educador externo en la disminución de las conductas de acoso escolar que se producían, fueron las entrevistas participantes (audios) entre cada acosador y el educador externo de la institución educativa, el registro de los tutores del centro que observaban las conductas bullying y las víctimas que las sufrían.

Para valorar la realidad de lo sucedido en las diferentes intervenciones, las actuaciones han sido documentadas, transcritas y analizadas de forma sistemática, no arbitraria e imparcial, atendiendo a criterios de adaptabilidad, variabilidad, gradualidad y pertenencia (Santos, 1990). Con el fin de extraer, codificar y categorizar todos los datos recogidos para su análisis cualitativo, y poder realizar un análisis descriptivo e interpretativo de la información, se ha utilizado la herramienta informática ATLAS.ti (versión 22), y se han transformado los datos en tablas y gráficas.

\section{Resultados}

El análisis de los datos de las respuestas que han dado los tres acosadores a lo largo de las seis sesiones que han durado las intervenciones, ha dado como resultado un sistema de categorías que se explica a continuación y que se recoge de forma sintética (tabla 1).

La primera macro-categoría identificada es la denominada situación del acosador y se refiere a la percepción que tienen los alumnos maltratadores respecto a la razón o causa por la que son interpelados por un educador externo al centro educativo. Esta macro-categoría emerge de la categoría función del educador, que se identifica a través de la descripción que realizan los acosadores sobre quién es la persona que les interpela y la razón por la que está con ellos; [...] eres un educador, $[\ldots]$ eres un inspector, $[\ldots]$ por lo que he hecho $[\ldots]$ para trabajar conmigo [...] para ayudarme. La categoría motivo de la situación también se asocia a la macro-categoría señalada y se observa cuando los estudiantes señalan y citan el nombre de la víctima directamente.

El hecho de que los agresores se responsabilicen de las conductas de acoso realizadas, en cambio, se asocia a la macro-categoría responsabilidad. En ella se incluye la categoría de 
reconocimiento de las conductas realizadas, que se identifica cuando los alumnos agresores hacen suyas las agresiones perpetradas; [...] la amenazo, [...] le obligo a que coma mi comida en el comedor, [...] le pego en clase, [...] le pego a su amiga si no hace lo que quiero, [...] le obligo a que se coma la comida que no me gusta, [...] le tiro gomas y tizas, etc.

Otra categoría perteneciente a la macro-categoría de responsabilidad es la de trascendencia de las conductas realizadas. Esta se aprecia cuando los acosadores asumen que las acciones que han realizado van a tener unas consecuencias; [...] te castigan, [...] te echan de la escuela.

La siguiente macro-categoría que se ha encontrado es la de reconocimiento social. Esta hace referencia al hecho de que los estudiantes se reconozcan como el sujeto que infringe daño a alguien. Esta macro-categoría aflora de las categorías de identificación del rol representado y de reconocerse como acosador. La primera se aprecia cuando los maltratadores asocian sus conductas agresivas con las de un acosador; [...] el que pega, [...] el que mete miedo, [...] el que molesta, [...] el que la lía. La segunda, en cambio, se observa cuando aceptan su condición de maltratador; [...] soy el agresor.

Otra de las macro-categorías encontradas, la de análisis, hace referencia al sentido que le dan los estudiantes a las conductas que han realizado. En ella se enmarcan dos categorías. La que se ha designado como conocimiento del tipo de agresión, que se observa cuando el alumno clasifica y enmarca las agresiones perpetradas como conductas que perjudican gravemente la convivencia en el centro docente, y la denominada valoración del tipo de agresión, que se aprecia cuando valora la gravedad del tipo de conductas que ha realizado.

La macro-categoría de evaluación, sin embargo, recoge la proyección que realizan los estudiantes de las consecuencias que puede tener realizar las conductas agresivas acosadoras. La comprensión de las consecuencias y la evaluación de ellas son las dos categorías que subyacen bajo ella. El sentido de la primera se vincula a la descripción por parte de los acosadores de las consecuencias que tiene acosar y se observa cuando las describe; [...] juicio, $[\ldots]$ no ir a clase, $[. .$.$] que no me dejan ir a clase, [...] expulsado, [...] mandarme, a otro centro. La$ categoría evaluación de las consecuencias por parte de los estudiantes pone el acento en el hecho de que los alumnos evalúen sus conductas y las consecuencias de estas. Esta categoría se hace presente en la medida en que estos predicen lo señalado; $[. .$.$] echarme, [\ldots]$ juicio, $[. .$. hacerme hacer, $[\ldots]$ trabajos en la escuela.

Por último, se ha encontrado la macro-categoría de consciencia. Esta se entiende como el reconocimiento que realiza el agresor de que no hay razón objetiva para agredir a la persona que acosa. Esta macro-categoría se compone de dos categorías. La primera, motivo del acoso, se aprecia cuando los acosadores relacionan las conductas de acoso realizadas con los objetivos que persiguen y sus motivos; [...] para que haga lo que yo diga, [...] a mi otras veces también me ha pasado, $[. .$.$] es chica, [. .$.$] yo siempre tengo que hacer todo lo que me dicen, aunque no quiera$ [...] mi hermano siempre me pega, si no hago lo que él dice, [...] mi padre le llama zorra... a mi madre, [...] siempre todo me pasa mí. La segunda categoría, despersonalización, hace referencia a que los estudiantes en su razonamiento del acto de agresión no involucran a la persona que agreden. Esta categoría se encuentra latente en el momento en que los acosadores verbalizan que su problema no es la persona a la que acosan; [...] mi familia, $[\ldots]$ mi padre, $[\ldots]$ mi hermano, [...] mi vida.

En el análisis categorial realizado se identifican doce momentos o situaciones concretas (categorías) y seis fases o estados por los que los agresores (macro-categorías) pasaron durante las sesiones antes de dejar de agredir, tal y como se muestra a continuación de forma resumida (ver tabla 1.). 
TABLA 1. Categorización de las fases y momentos por las que pasan los agresores antes de dejar de agredir

\begin{tabular}{lllc}
\hline \multicolumn{2}{c}{$\begin{array}{c}\text { Macrocategorías } \\
\text { Fases }\end{array}$} & \multicolumn{1}{c}{$\begin{array}{c}\text { Categorías } \\
\text { Momentos }\end{array}$} & Sesiones \\
\hline A & Situación & $\begin{array}{l}\text { Función del educador } \\
\text { Motivo de la situación }\end{array}$ & 1 \\
B & Responsabilidad & $\begin{array}{l}\text { Reconocimiento de las conductas realizadas } \\
\text { Trascendencia de la conductas realizadas }\end{array}$ & 2 \\
C Reconocimiento social & $\begin{array}{l}\text { Identificación del rol representado } \\
\text { Reconocerse como acosador } \\
\text { Conocimiento del tipo de agresión } \\
\text { Valoración del tipo de agresión }\end{array}$ & 3 \\
D Análisis & $\begin{array}{l}\text { Comprensión de las consecuencias } \\
\text { Evaluación de las consecuencias hacia él }\end{array}$ & 4 \\
E Evaluación & $\begin{array}{l}\text { Motivo del acoso } \\
\text { Despersonalización }\end{array}$ & 5 \\
\hline
\end{tabular}

Fuente: elaboración propia

A través del instrumento de análisis cualitativo Atlas/ti se ha trasformado la categorización realizada en una representación (figura 1) de la que se extrae que el conjunto de fases o estados que se han provocado en el sujeto acosador durante las intervenciones, contribuyen a detener las conductas de acoso.

\section{FIGURA 1. Estados que contribuyen a la detención de las conductas de acoso}

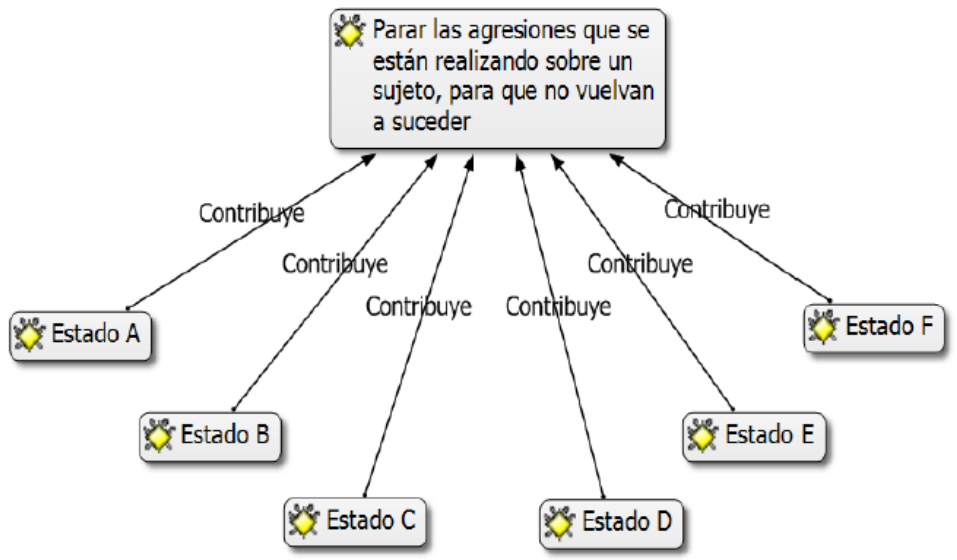

Fuente: Atlas/ti

Un análisis más profundo de la información recogida de las entrevistas participantes (audios) con los acosadores, el registro de los tutores del centro y las víctimas, indica que las intervenciones realizadas por el educador en las diferentes intervenciones contribuyen a una progresiva desaparición de las conductas de acoso (figura 2). 
FIGURA 2. Evolución de las conductas de acoso de los tres agresores

\begin{tabular}{|c|c|c|c|c|c|c|c|}
\hline \multicolumn{2}{|c|}{$\begin{array}{l}\text { Conductas de acosoo } \\
\text { Conductas de acoso extinguidas }\end{array}$} & \multicolumn{6}{|c|}{ Intervenciones } \\
\hline & Acosador & 1 & 2 & 3 & 4 & 5 & 6 \\
\hline Amenazas verbales & $\begin{array}{l}1 \\
2 \\
3\end{array}$ & $\begin{array}{l}0 \\
0 \\
0\end{array}$ & $\begin{array}{l}0 \\
0 \\
0\end{array}$ & $\begin{array}{l}0 \\
0 \\
0\end{array}$ & $\begin{array}{l}0 \\
0 \\
0\end{array}$ & $\begin{array}{l}0 \\
0 \\
0\end{array}$ & $\begin{array}{l}0 \\
0 \\
0\end{array}$ \\
\hline Amenazas gestuales & $\begin{array}{l}1 \\
2 \\
3\end{array}$ & $\begin{array}{l}0 \\
0 \\
0\end{array}$ & $\begin{array}{l}0 \\
0 \\
0\end{array}$ & $\begin{array}{l}0 \\
0 \\
0\end{array}$ & $\begin{array}{l}0 \\
0 \\
0\end{array}$ & $\begin{array}{l}0 \\
0 \\
0\end{array}$ & $\begin{array}{l}0 \\
0 \\
0\end{array}$ \\
\hline Amenaza con agresión física & $\begin{array}{l}1 \\
2 \\
3\end{array}$ & $\begin{array}{l}0 \\
0 \\
0\end{array}$ & $\begin{array}{l}0 \\
0 \\
0\end{array}$ & $\begin{array}{l}0 \\
0 \\
0\end{array}$ & $\begin{array}{l}0 \\
0 \\
0\end{array}$ & $\begin{array}{l}0 \\
0 \\
0\end{array}$ & $\begin{array}{l}0 \\
0 \\
0\end{array}$ \\
\hline Chantaje con amenazas & $\begin{array}{l}1 \\
2 \\
3\end{array}$ & $\begin{array}{l}0 \\
0 \\
0\end{array}$ & $\begin{array}{l}0 \\
0 \\
0\end{array}$ & $\begin{array}{l}0 \\
0 \\
0\end{array}$ & $\begin{array}{l}0 \\
0 \\
0\end{array}$ & $\begin{array}{l}0 \\
0 \\
0\end{array}$ & $\begin{array}{l}0 \\
0 \\
0\end{array}$ \\
\hline Agresiones físicas & $\begin{array}{l}1 \\
2 \\
3\end{array}$ & $\begin{array}{l}0 \\
0 \\
0\end{array}$ & $\begin{array}{l}0 \\
0 \\
0\end{array}$ & $\begin{array}{l}0 \\
0 \\
0\end{array}$ & $\begin{array}{l}0 \\
0 \\
0\end{array}$ & $\begin{array}{l}0 \\
0 \\
0\end{array}$ & $\begin{array}{l}0 \\
0 \\
0\end{array}$ \\
\hline $\begin{array}{l}\text { Coportamientos disruptivos } \\
\text { hacia la víctima }\end{array}$ & $\begin{array}{l}1 \\
2 \\
3\end{array}$ & $\begin{array}{l}0 \\
0 \\
0\end{array}$ & $\begin{array}{l}0 \\
0 \\
0\end{array}$ & $\begin{array}{l}0 \\
0 \\
0\end{array}$ & $\begin{array}{l}0 \\
0 \\
0\end{array}$ & $\begin{array}{l}0 \\
0 \\
0\end{array}$ & $\begin{array}{l}0 \\
0 \\
0\end{array}$ \\
\hline Acoso social & $\begin{array}{l}1 \\
2 \\
3\end{array}$ & $\begin{array}{l}0 \\
0 \\
0\end{array}$ & $\begin{array}{l}0 \\
0 \\
0\end{array}$ & $\begin{array}{l}0 \\
0 \\
0\end{array}$ & $\begin{array}{l}0 \\
0 \\
0\end{array}$ & $\begin{array}{l}0 \\
0 \\
0\end{array}$ & $\begin{array}{l}0 \\
0 \\
0\end{array}$ \\
\hline Insultos & $\begin{array}{l}1 \\
2 \\
3\end{array}$ & $\begin{array}{l}0 \\
0 \\
0\end{array}$ & $\begin{array}{l}0 \\
0 \\
0\end{array}$ & $\begin{array}{l}0 \\
0 \\
0\end{array}$ & $\begin{array}{l}0 \\
0 \\
0\end{array}$ & $\begin{array}{l}0 \\
0 \\
0\end{array}$ & $\begin{array}{l}0 \\
0 \\
0\end{array}$ \\
\hline Robos & $\begin{array}{l}1 \\
2 \\
3\end{array}$ & $\begin{array}{l}0 \\
0 \\
0\end{array}$ & $\begin{array}{l}0 \\
0 \\
0\end{array}$ & $\begin{array}{l}0 \\
0 \\
0\end{array}$ & $\begin{array}{l}0 \\
0 \\
0\end{array}$ & $\begin{array}{l}0 \\
0 \\
0\end{array} \mid$ & $\begin{array}{l}0 \\
0 \\
0\end{array}$ \\
\hline
\end{tabular}

Fuente: elaboración propia

Por último, el estudio pone de manifiesto que los comportamientos más visibles, las agresiones físicas o las amenazas verbales, se extinguen antes que las que son de carácter más oculto o tendencia sibilina, como las amenazas gestuales o los comportamientos disruptivos hacia la víctima.

\section{Conclusiones}

Una vez realizado el estudio de los datos obtenidos de las intervenciones del educador con los alumnos acosadores, se concluye que los tres estudiantes acosadores han pasado por diferentes momentos y fases antes de dejar de realizar bullying a sus víctimas. 
Del análisis de las diferentes intervenciones se observa como en la primera fase los acosadores conocen la función y el motivo por el que se encuentran con el educador externo, lo que les dispone a un estado de alerta o situación de vigilia. Al comienzo de las intervenciones los estudiantes también perciben la responsabilidad que tienen, ya que reconocen las conductas agresivas realizadas y su trascendencia. Es decir, asumen que las acciones que han realizado tienen un significado negativo.

En la segunda fase los estudiantes reconocen socialmente sus comportamientos agresivos. Identifican sus conductas con las del rol del agresor y se reconocen e identifican como acosadores. Estos dos momentos son de especial relevancia porque la atribución causal interna de los acontecimientos que realizan contribuye a disminuir las conductas agresivas (Dykeman, Daehlin, Doyle y Flamer, 1996; Wallace, Barry, Zeigler-Hill y Green, 2012).

En la tercera fase los maltratadores conocen y valoran la gravedad el tipo de conducta que han realizado. Este análisis les ayuda a recodificar el tipo de conductas que han cometido y a contextualizar lo que han hecho.

En la siguiente fase los estudiantes comprenden y evalúan las consecuencias que va a tener el acoso que han realizado, lo que les aproxima a experimentar el resultado negativo de haber quebrado las normas de convivencia (Suckling y Temple, 2006) y a desarrollar el sentido de las consecuencias (Antelo, Cagide, Cruz y Buceta, 2010), es decir evalúan el costo de respuesta de sus acciones.

En las dos últimas fases los acosadores son conscientes del motivo por el que han acosado, debido a que trabajan las habilidades de pensamiento de tipo causal, alternativo y consecuencial que propone Segura (2005). Estos relacionan las conductas de acoso realizadas con los objetivos que persiguen, y por otra parte con sus motivos, es decir, diferencian el para qué han agredido del por qué han agredido. Fundamentalmente los acosadores se hacen conscientes y despersonalizan a la víctima de los motivos por los que agreden. Al no encontrar ninguna razón objetiva para responder de forma agresiva sobre sus víctimas dejan de agredir. La consciencia proporciona la libertad de decisión sobre a las conductas que se desean llevar a cabo.

De la investigación se concluye que el diseño de un esquema de actuación que esté compuesto por una actividad cognitiva y un ejercicio razonamiento que tenga como metas secuenciadas que el agresor conozca la situación en la se encuentra, se responsabilice de lo que ha hecho, reconozca socialmente que ha agredido, analice y valore las conductas que ha realizado, y sea consciente de por qué agrede, facilita que deje de agredir.

Teniendo en cuenta la detección de la desaparición progresiva de las conductas agresivas en los tres estudiantes, también se concluye que la planificación de un conjunto de intervenciones que contribuye a que los agresores conozcan la función que tiene el educador externo y el motivo por el que se encuentra con él, así como que reconozcan las conductas realizadas y la trascendencia que tienen, identifiquen el rol que han representado, reconozcan que son acosadores, conozcan y valoren el tipo de agresión que han realizado, además de que comprendan y evalúen las consecuencias de su conducta, entienda el motivo por el que han acosado y lo despersonalicen de éste, detienen las conductas de acoso escolar progresivamente.

Estos dos diseños de actuación que se proponen como conclusión en este trabajo, y que se derivan de la categorización realizada, guardan la suficiente lógica, coherencia y flexibilidad como para poder ser aplicadas en diferentes contextos y servir de ayuda a los profesionales de la educación y a las instituciones educativas que se encuentren con estas situaciones en su práctica docente. $Y$ esto es así fundamentalmente porque el diseño de las actividades cognitivas y ejercicios de razonamiento puede ser cualquiera mientras respondan o contribuyan al desarrollo de las fases o momentos señalados. 
De la investigación realizada también se concluye que antes de intervenir es importante identificar al ideólogo de las agresiones, al motivador primario, para apartarlo de los acosadores gregarios. El hecho de orientar las actuaciones hacia el maltratador precursor, el líder, favorece que se individualice la responsabilidad sobre él y contribuye a que muestre más interés por dejar de acosar.

Otra de las cuestiones que repercute en la detención de las conductas de acoso es el interés que muestra el educador sobre el agresor, ya que agredir significa menoscabar la confianza de éste e ir en contra de la persona que trata de ayudarle para que deje de acosar y no se abra el protocolo de actuación antibullying. En este sentido es imprescindible entender que las sanciones por sí solas no detienen el bullying.

A la hora de realizar este trabajo entre las limitaciones previas se encuentra la forma en que los centros educativos conciben el acoso escolar, ya que pretenden responder al problema por medio de recetas y desde una perspectiva cortoplacista y no sistémica, es decir de forma rápida, discreta y sin ser molestado.

Respecto a las futuras líneas de investigación, estas deberían encaminarse hacia el diseño de instrumentos específicos que permitiesen identificar a los tipos de acosador según sus motivaciones para responder de forma concreta por medio de un procedimiento personalizado. Estos recursos deberían estar a disposición de los centros para poder implementarlo en el momento en el que lo necesiten.

\section{Referencias bibliográficas}

Álvarez-García, D., Núñez, J.C., Rodríguez, C., Álvarez, L. y Dobarro, A. (2011). Propiedades psicométricas del Cuestionario de Violencia Escolar Revisado (CUVE-R). Revista de Psicodidáctica, 16(1), 59-83. doi: http://dx.doi.org/10.1387/RevPsicodidact.1146

Andreu, J.M., Peña, M.E. y Ramírez, J.M. (2009). Cuestionario de agresión reactiva y proactiva un instrumento de medida de la agresión en adolescentes. Revista de Psicopatología y Psicología Clínica, 14(1), 37-49. doi: http://dx.doi.org/10.5944/rppc.vol.14.num.1.2009.4065

Andreu, J.M., Ramirez, J.M. y Raine, A. (2006). Un modelo dicotómico de la agresión: valoración mediante dos autoinformes (CAMA y RPQ). Psicopatología Clínica, Legal y Forense, 5, 25-42.

Antelo, Y., Cagide, M.E., Cruz, V. y Buceta, M. J. (2010). Qué se puede hacer para trabajar con niños que acosan a sus compañeros. En J.J. Gázquez y M.C. Pérez (Coords.). La convivencia escolar: aspectos psicológicos y educativos, 199-204. Granada: Grupo Editorial Universitario.

Arsenio, W.F., Gold, J, y Adams, E. (2004). Adolescents'emotion expectancies regarding aggressive and nonaggressive events: connections with behavior problems. Journal of Experimental Child Psychology: special emotional cognition in children, 89, 338-355. doi: doi:10.1016/j.jecp.2004.08.001

Avilés, J. M. (2013). Bullying: el maltrato entre iguales. Agresores. Víctimas y testigos en la escuela. Salamanca: Amarú.

Avilés, J.M. (2006). Diferencias de atribución causal en el bullying entre sus protagonistas, Revista electrónica de investigación psicoeducativa, 9 (4), 201-220. doi: http://dx.doi.org/10.14204/ejrep.38.14123 
Beane, A.L. (2006). Aulas libres de acoso. Barcelona: Graó.

Bender, D. y Lösel, F. (2011). Bullying at school as a predictor of delinquency, violence and other anti- social behaviour in adulthood. Criminal behaviour and mental health, 21(2), 99-106. doi: 10.1002/cbm.799

Berger, C. y Caravita, C.S. (2016). Why do Early Adolescents Bully? Exploring the Influence of Prestige Norms on Social and Psychological Motives to Bully. Journal of Adolescence, 46, 4556. doi: http://dx.doi.org/10.1016/j.adolescence.2015.10.020

Berger, K. S. (2006). Psicología del Desarrollo Infancia y Adolescencia. Madrid: Editorial Médico Panamericana.

Bush, C.A., Mullis, R.L. y Mullis, A.K. (2000). Differences in empathy between offender and nonoffender youth. Journal of Youth and Adolescence, 29, 467-478. doi: 10.1023/A:1005162526769

Cassiani-Miranda, C. A., Gómez-Alhach, J., Cubides-Munévar, A. M. y Hernández-Carrillo, M. (2014). Prevalencia de bullying y factores relacionados en estudiantes de bachillerato de una institución educativa de Cali, Colombia, 2011. Revista de salud pública, 16(1), 13-24. doi: Http://Dx.Doi.Org/10.15446/Rsap.V16n1.43490

Cerezo, F. (2006). Violencia y victimización entre escolares. El bullying: estrategias de identificación y elementos para la intervención a través del Test Bull-S. Electronic journal of research in educational psychology, 4(9), 333-352.

Cerezo, F. (2009). Bullying: Análisis de la situación en las aulas españolas. International Journal of Psychology and Psychological Therapy, 9(3), 383-395.

Cerezo, F., Calvo, A.R. y Sánchez, C. (2011). El programa CIP para la intervención específica en bullying. Madrid: Pirámide.

Cerezo, F. y Méndez, I. (2013). Agresores en bullying y conductas antisociales. European Journal of Investigation in Health, Psychology and Education, 3(1), 5-14.

Coover, G.E. y Murphy, S.T. (2000). The communicated self: Exploring the interaction between self and social context. Human Communication Research, 26, 125-147. doi: 10.1111/j.14682958.2000.tb00753.x

Della Cioppa, V., O'neil, A. y Craig, W. (2015). Learning from traditional bullying interventions: A review of research on cyberbullying and best practice. Aggression and Violent Behavior, 23, 61-68. doi: http://dx.doi.org/10.1016/j.avb.2015.05.009

Díaz-Aguado, M.J. (2004). Prevención de la violencia y lucha contra la exclusión desde la adolescencia. La violencia entre iguales en la escuela y en el ocio. Volumen 2: Programa de intervención y estudio experimental. Madrid: Instituto de la Juventud. Ministerio de Trabajo y Asuntos Sociales.

Dueñas Buey, M. y Senra, M. (2009). Habilidades sociales y acoso escolar: Un estudio en centros de enseñanza secundaria de Madrid. Revista Española de Orientación y Psicopedagogía, REOP, 1(20), 39-49.

Dykeman, C., Daehlin, W., Doyle, S. y Flamer, H.S. (1996). Psychological Predictors of SchoolBased Violence: Implications for School Counselors. School Counselor, 44(1), 35-47.

Einarsen, S. y Hauge, L. (2006). Antecedentes y consecuencias del acoso psicológico en el trabajo: una revisión de la literatura. Revista de Psicología del Trabajo y de las Organizaciones, 22(3), 251-274.

Elliot, J. (1993). El cambio educativo desde la investigación-acción. Madrid: Morata. 
Espelage, D.L. y Swearer, S.M. (2003). Research on School Bullying and Victimization: What Have We Learned and Where Do We Go From Here? School Psychology Review, 32(3), 365-383.

Estévez, E., Herrero, J., Martínez, B. y Musitu, G. (2006). Aggressive and non-aggressive rejected students: An analysis of their differences. Psychology in the Schools, 43, 387-400. doi: http://dx.doi.org/10.1002/pits.20152

Ferrel, F., Cuan, A., Londoño, Z. y Ferrel, L. (2015). Factores de riesgo y protectores del bullying escolar en estudiantes con bajo rendimiento de cinco instituciones educativas de Santa Marta, Colombia. Psicogente, 18(33), 188-205. doi: http://doi.org/10.17081/psico.18.33.65

Frick, P. J., Cornell, A.H., Bodin, S. D., Dane, H. A., Barry, C.T. y Loney, B. R. (2003). Callousunemotional traits and developmental pathways to severe conduct problems. Developmental Psychology, 39, 246-260. doi: http://dx.doi.org/10.1037/0012-1649.39.2.246

Garaigordobil, M. y Oñederra, J.A. (2010a). La violencia entre iguales. Madrid: Pirámide.

Garaigordobil, M. y Oñederra, J.A. (2010b). Inteligencia emocional en las víctimas de acoso escolar y en los agresores. European Journal of Education and Psychology, 3(2), 243-256. doi: 10.1989/ejep.v3i2.63

Gázquez, J. J., Pérez, M. C. y Carrión, J. J. (2011). Clima escolar y resolución de conflictos según el alumnado: un estudio europeo. Revista de Psicodidáctica, 16, 39-58. doi:10.1387/RevPsicodidact.1144.

Gini, G., Pozzoli, T. y Hauser, M. (2011). Bullies have Enhanced Moral Competence to Judge relative to Victims, but Lack Moral Compassion. Personality and Individual Differences, 50, 603-608. doi: http://dx.doi.org/10.1016/j.paid.2010.12.002

Gobierno Vasco. Decreto 201/2008, de 2 de diciembre sobre derechos y deberes de los alumnos y alumnas de los centros docentes no Universitarios de la Comunidad Autónoma del País Vasco. Boletín oficial del País Vasco, 16 de diciembre de 2008, №240, (30963).

Gómez, A., Gala, F. J., Lupiani, M., Bernalte, A., Miret, M. T., Lupiani, S., y Barreto, M. C. (2005). El "bullying" y otras formas de violencia adolescente. Cuadernos de Medicina Forense 13(4849), 165-177.

González-Ortega, I., Echeburúa, E. y Corral, P. (2008). Variables significativas en las relaciones violentas en parejas jóvenes: una revisión. Psicología Conductual, 16(2), 207-225.

Greenberg, M. T. (2010). School-based prevention: current status and future challenges. Effective Education, 2(1), 27-52. doi: 10.1080/19415531003616862

Gutiérrez, M. Escartí, A. y Pascual, C. (2011). Relaciones entre empatía, conducta prosocial, agresividad, autoeficacia y responsabilidad personal y social de los escolares. Psicothema, 23(1), 13-19.

Hanish, L. y Guerra, N.G. (2004). Aggressive victims, passive victims, and bullies: developmental continuity or developmental change? Merrill Palmer Quarterly, 50(1), 17-38. doi: http://dx.doi.org/10.1353/mpq.2004.0003

Hawley, P. H., Stump, K. N. y Ratliff, J. M. (2010). Side stepping the jingle fallacy: Bullying, aggression, and the importance of knowing the difference. En D. Espelage y S. Swearer (Eds.), Bullying in American schools (2ed.), 101-115. New York: Rutledge.

Hubbard, J. A., Dodge, K. A., Cillessen, A. H. N., Coie, J. D. y Schwartz, D. (2001). The dyadic Nature of social information processing in boys reactive and proactive aggression. Journal of Personality and Social Psychology, 80, 268-280. doi: http://dx.doi.org/10.1037//00223514.80.2.268 
Hubbard, J. A., Mcauliffe, M. D., Morrow, M. T. y Romano, L. J. (2010). Reactive and proactive aggression in childhood and adolescence: Precursors, outcomes, processes, experiences and measurement. Journal of Personality, 78, 95-118.

Jiménez-Barbero, J. A., Ruiz-Hernández, J. A., Velandrino-Nicolás, A. P. y Llor-Zaragoza, L. (2016). Actitudes hacia la violencia, impulsividad, estilos parentales y conducta externalizada en adolescentes: comparación entre una muestra de población general y una muestra clínica. Anales de psicología, 32(1), 132-138. doi: http://dx.doi.org/10.6018/analesps.32.1.195091

Kernis, M.H., Grannemman, B.D. y Barclay, L.C. (1989). Stability and level of Self-Esteem as predictors of anger arousal and hostility. Journal of Personality and Social Psychology, 56, 1013-1022. doi: http://dx.doi.org/10.1037/0022-3514.56.6.1013

López, A., Domínguez, J. y Álvarez, E. (2010). Bullying vertical: variables predictivas de la violencia escolar. Revista de Investigación en Educación, 8, 24-38.

Marsee, M. A. y Frick, P. J. (2007). Exploring the cognitive and emotional correlates to proactive and reactive aggression in a sample of detained girls. Journal of Abnormal Child Psychology, 35, 969-981. doi: http://dx.doi.org/10.1007/s10802-007-9147-y

Martínez, B., Buelga, S. y Cava, M.J. (2007). La satisfacción con la vida en la adolescencia y su relación con la autoestima y el ajuste escolar. Anuario de Psicología, 38(2), 5-15.

Martorell, C., González, R., Rasal, P. y Estellés, R. (2009). Convivencia e inteligencia emocional en niños en edad escolar. European Journal of Education and Psychology, 2(1), 69-78

Mckernan, J. (1999). Investigación-acción y currículum. Madrid: Morata.

Mestre, V., Samper, P., Tur-Porcar, A. M., Richaud De Minzi, M. C. y Mesurado, B. (2012). Emociones, estilos de afrontamiento y agresividad en la adolescencia. Universitas Psychologica, 11, 1263-1275.

Olweus, D. (1993). Bullying At School: What We Know and What We Can Do. Oxford: Blackwell.

Ortega, R. y Mora-Merchán, J. A. (2008). Las redes de iguales y el fenómeno del acoso escolar: Explorando El Esquema Dominio-Sumisión. Infancia y aprendizaje, 31(4), 515-528. Doi: http://dx.doi.org/10.1174/021037008786140922

Pelegrín, A. y Garcés de los Fayos, E.J. (2008). Variables contextuales y personales que inciden en el comportamiento violento del niño. European Journal of Education and Psychology, 1(1), 5-20.

Perren, S. y Alsaker, F. D. (2006). Social behavior and peer relationships of victims, bully- victims, and bullies in kindergarten. Journal of child psychology and psychiatry, 47(1), 45-57. doi: 10.1111/j.1469-7610.2005.01445.x

Piñuel, I. y Oñate, A. (2007). Mobbing escolar: Violencia y acoso psicológico contra los niños. Barcelona: CEAC.

Rodkin, P., Ryan, A., Jamison R. y Wilson T. (2013). Social Goals, Social Behavior, and Social Status in Middle Childhood. Developmental Psychology, 49, 1.139-1.150. doi: http://dx.doi.org/10.1037/a0029389

Romera, E. M., Cano, J. J., García-Fernández, C. M. y Ortega-Ruiz, R. (2016). Cyberbullying: competencia social, motivación y relaciones entre iguales. Comunicar: Revista Científica de Comunicación y Educación, 24(48). doi: http://dx.doi.org/10.3916/C48-2016-07

Romero, L. L., Triñanes, E. R. y Iglesias, B. G. (2011). Delimitando la agresión adolescente: Estudio diferencial de los patrones de agresión reactiva y proactiva. Revista Española De Investigación Criminológica, REIC, 9, 2-29.

Santos, M. A. (1990). Hacer visible lo cotidiano. Madrid: Akal. 
Segura, M. (2005). Enseñar a convivir no es tan difícil. Bilbao: Desclée de Brouwer.

Smithmyer, C.M., Hubbard, J.A. y Simons, R.F. (2000). Proactive and reactive aggression in delinquent adolescents: relations to aggression outcome expectancies. Journal of Clinical Child Psychology, 29, 86 - 93. doi: http://dx.doi.org/10.1207/s15374424jccp2901_9

Suckling, A. y Temple, C. (2006). Herramientas contra el acoso escolar. Un enfoque integral. Madrid: Morata.

Valdés Cuervo, A. A., Yañez Quijada, A. I. y Martínez, E. A. C. (2013). Diferencias entre subgrupos de estudiantes involucrados en el bullying: víctimas, agresores-víctimas y agresores. Liberabit, 19(2), 215-222.

Vera, R. (2010). Violencia en las aulas: el Bullying o acoso escolar. Innovación y Experiencias Educativas, 37, 1-9.

Vitaro, F., Brendgen, M. y Tremblay, R.E. (2002). Reactively and proactively aggressive children: antecedent and subsequent characteristics. Journal of Child and Psychology Psychiatry, 43 (4), 495-505. doi: http://dx.doi.org/10.1111/1469-7610.00040

Vreeman, R.C. y Carroll, A.E. (2007). A systematic review of school-based interventions to prevent bullying. Archives of Pediatrics and Adolescent Medicine, 161, 78-88. doi: http://dx.doi.org/10.1001/archpedi.161.1.78

Wallace, M. T., Barry, C. T., Zeigler Hill, V. y Green, B. A. (2012). Locus of control as a contributing factor in the relation between self- perception and adolescent aggression. Aggressive Behavior, 38(3), 213-221. doi: 10.1002/ab.21419

Weare, K. y Nind, M. (2011). Mental health promotion and problem prevention in schools: what does the evidence say? Health Promotion International 26(1), 129-169. doi: http://dx.doi.org/10.1093/heapro/dar075

Zimmerman, F.J. (2005). Early cognitive stimulation, emotional support, and television watching as predictors of subsequent bullying among grade-school children. Archives of Pediatrics $y$ Adolescent Medicine, 159(4), 384-388. doi: http://dx.doi.org/10.1001/archpedi.159.4.384

Zych, I., Ortega-Ruiz, R. y Del Rey, R. (2015). Scientific research on bullying and cyberbullying: Where have we been and where are we going. Aggression and Violent Behavior, 24, 188-198. doi: http://dx.doi.org/10.1016/j.avb.2015.05.01

Fecha de entrada: 18 Marzo 2017

Fecha de revisión: 26 Febrero 2018 Fecha de aceptación: 1 Marzo 2018 